\title{
Hierarchy of structure functions for passive scalars advected by turbulent flows
}

\author{
Guowei He ${ }^{\mathrm{a}, \mathrm{b}}$, Shiyi Chen ${ }^{\mathrm{a}}$, Gary Doolen ${ }^{\mathrm{a}}$ \\ a Theoretical Division and Center for Nonlinear Studies, Los Alamos National Laboratory, Los Alamos. NM 87545, USA \\ ${ }^{b}$ LNM, Institute of Mechanics, Chinese Academy of Sciences, Beijing, 100080, China
}

Received 22 April 1998; accepted for publication 19 June 1998

Communicated by A.R. Bishop

\begin{abstract}
A hierarchical model is proposed for the joint moments of the passive scalar dissipation and the velocity dissipation in fluid turbulence. This model predicts that the joint probability density function (PDF) of the dissipations is a bivariate $\log$-Poisson. An analytical calculation of the scaling exponents of structure functions of the passive scalar is carried out for this hierarchical model, showing a good agreement with the results of direct numerical simulations and experiments. (C) 1998 Published by Elsevier Science B.V.
\end{abstract}

PACS: $05.60 .+\mathrm{w} ; 47.27 .-\mathrm{i} ; 47.27 . \mathrm{Gs}$

The transport of a passive scalar field such as temperature, $T$, is governed by the advection-diffusion equation,

$\frac{\partial T}{\partial t}+(\boldsymbol{u} \cdot \nabla) T=D \triangle T$,

where $D$ is the diffusion coefficient, and $\boldsymbol{u}$ is the solenoidal velocity field governed by the NavierStokes equations,

$\frac{\partial \boldsymbol{u}}{\partial t}+(\boldsymbol{u} \cdot \nabla) \boldsymbol{u}=-\nabla P+\nu \Delta \boldsymbol{v}+\boldsymbol{f}$.

Here $f$ is the external force, $P$ is the pressure and $\nu$ is the kinematic viscosity. In the stationary unforced inviscid and nondiffusive limit $(\nu, D \rightarrow 0)$, (1) and (2) are invariant under a scale transformation: $(t, u, x, T) \rightarrow\left(\lambda^{-(1-h)} t, \lambda^{-1} x, \lambda^{h} u, \lambda^{h} T\right)$, where $\boldsymbol{x}$ is the spatial coordinate vector, $\lambda$ is an arbitrary positive real number and $h$ is an arbitrary real num- ber. For the inertial-convective subrange where the velocity is no influence of viscosity and the scalar is simply transported by the velocity field [1], this scale invariance implies that the structure functions for the velocity and for the passive scalars obey power laws. Dimensional analysis suggests that $n$ th-order structure functions of velocity and passive scalars obey the following linear scaling relations [2-4],

$$
\left\langle\left(\delta u_{\ell}\right)^{n}\right\rangle \sim \ell^{n / 3}, \quad\left\langle\left(\delta T_{\ell}\right)^{n}\right\rangle \sim \ell^{n / 3},
$$

where $\delta u_{\ell} \equiv \boldsymbol{u}(x+\ell)-\boldsymbol{u}(x)$ is the longitudinal velocity increment, $\delta T_{\ell} \equiv T(x+\ell)-T(x)$ is the scalar increment and $\ell$ is the spatial separation in the inertial subrange. However, existing experiments [5-7] and numerical simulations $[4,8,9]$ do not support these predictions. The possible cause for the deviations from the predictions is believed to be associated with intermittencies of velocity and scalar dissipations. The 
observations also indicate that the scalar dissipation exhibits higher intermittency than the velocity dissipation, leading to larger deviations from the linear scaling exponents for passive scalars than for velocity [7].

To take account of intermittency corrections to the dimensional analysis, Kolmogorov proposed the refined similarity hypothesis (RSH) [10] for the velocity field

$\delta u_{\ell}=w_{u}\left(\epsilon_{\ell} \ell\right)^{1 / 3}$.

Obukhov and Corrsin proposed a similar RSH for the passive scalar field $[3,11]$,

$\delta T_{\ell}=w_{T} \ell^{1 / 3} \epsilon_{\ell}^{-1 / 6} N_{\ell}^{1 / 2}$.

Here $w_{u}$ and $w_{T}$ are random variables whose statistics only depend on the Reynolds number. $\epsilon_{\ell}$ and $N_{\ell}$ are the locally averaged velocity dissipation and scalar dissipation over a sphere of radius $\ell$, respectively. As seen from (4) and (5), the structure functions for velocity depend only on the properties of local averaged velocity dissipation, while the structure functions of passive scalars depend on the joint moments of both $\epsilon_{\ell}$ and $N_{\ell}$. In other words, in order to calculate the structure functions of the passive scalar, the joint probability density functions (PDF) of $\epsilon_{\ell}$ and $N_{\ell}$ are needed. In general, the joint PDF is more difficult to obtain than its marginal distribution. A simpler working approach is to infer the joint PDF from its marginal distribution. For example, because of the log-normality of $\epsilon_{\ell}$, the joint PDF of $\epsilon_{\ell}$ and $N_{\ell}$ can be inferred to be a bivariate log-normal distribution [12].

Recently, She and Leveque [13] have suggested using the log-Poisson PDF to describe $\epsilon_{\ell}$ on the basis of hierarchical structures. Cao and Chen [14] have extended this hypothesis and assumed a bivariate logPoisson distribution for the joint PDF of $\epsilon_{\ell}$ and $N_{\ell}$. Their model is in remarkable agreement with the results of numerical simulations and experiments. However, it has been not understood why $\epsilon_{\ell}$ and $N_{\ell}$ obey a bivariable log-Poisson distribution. In this brief report, we propose a hierarchical relation for the joint moments of both $\epsilon_{\ell}$ and $T_{\ell}$, and explain why hierarchical structures of joint moments lead to a bivariate $\log$-Poisson PDF for $\epsilon_{\ell}$ and $T_{\ell}$.

The intensity of the $p$ th-order dissipation structure can be characterized by the ratio of the successive moments of the turbulent dissipation, $\epsilon_{\ell}(p)=$ $\left\langle\epsilon_{\ell}^{p+1}\right\rangle /\left\langle\epsilon_{\ell}^{p}\right\rangle$. The ratios, $\epsilon_{\ell}(p)$, are assumed to obey the following hierarchical relations [13],

$\epsilon_{\ell}(p+1)=A_{p} \epsilon_{\ell}^{\beta}(p) \epsilon_{\ell}^{1-\beta}(\infty)$,

where $\epsilon_{\ell}(\infty) \equiv \lim _{p \rightarrow \infty} \epsilon_{\ell}(p)$ is associated with the most intermittent structure of turbulence; $A_{p}$ are functions which only depend on $p$; and $\beta$ is assumed to be a universal constant. Eq. (6) connects the ratios of successive dissipation moments with the most intermittent structures.

Similarly, one can characterize the intensity of the $q$ th-order structure of the scalar dissipation using the ratio of the successive moments of the scalar dissipation, $N_{\ell}(q)=\left\langle N_{\ell}^{q+1}\right\rangle /\left\langle N_{\ell}^{q}\right\rangle$. The qth-order ratio, $N_{\ell}(q)$, can be postulated to satisfy a hierarchical model similar to $\epsilon_{\ell}$, and the scalings of $N_{\ell}^{q}$ can be obtained from the hierarchical relations. However, this does not solve our problem since we require the scaling behavior of the joint moments of $N_{\ell}^{q}$ and $\epsilon_{\ell}^{p}$.

It has been observed from numerical simulations that the iso-surfaces of higher intensive passive scalars form sheets $[8,9]$, while the iso-surfaces of higher intensive vortices form filaments [15]. These higher intensive structures are strongly correlated. Therefore, it is reasonable to assume that $\left\langle\epsilon_{\ell}^{p} N_{\ell}^{q}\right\rangle \neq 0$. We denote the ratios of the successive joint moments by

$$
\begin{aligned}
& M_{\ell}(q, p)=\frac{\left\langle N_{\ell}^{q+1} \epsilon_{\ell}^{p}\right\rangle}{\left\langle N_{\ell}^{q} \epsilon_{\ell}^{p}\right\rangle}, \\
& \tilde{M}_{\ell}(q, p)=\frac{\left\langle N_{\ell}^{q} \epsilon_{\ell}^{p+1}\right\rangle}{\left\langle N_{\ell}^{q} \epsilon_{\ell}^{p}\right\rangle} .
\end{aligned}
$$

Now we postulate further that $M_{\ell}(q, p)$ and $\tilde{M}_{\ell}(q, p)$ obey the following extended hierarchical relations,

$$
\begin{aligned}
& M_{\ell}(q+1, p)=B_{q, p} M_{\ell}^{\beta_{T}}(q, p) M_{\ell}^{1-\beta_{T}}(\infty), \\
& \tilde{M}_{\ell}(q, p+1)=\tilde{B}_{q, p} \tilde{M}_{\ell}^{\beta}(q, p) \tilde{M}_{\ell}^{1-\beta}(\infty),
\end{aligned}
$$

where $M_{\ell}(\infty) \equiv \lim _{p, q \rightarrow \infty} M_{\ell}(q, p)$ and $\tilde{M}_{\ell}(\infty) \equiv$ $\lim _{p, q \rightarrow \infty} \tilde{M}_{\ell}(q, p)$ are the limits of the sequences of ratios of the successive joint moments; $B_{q, p}$ and $\tilde{B}_{q, p}$ are functions independent of the scale $\ell$; and $\beta_{T}$ and $\beta$ are universal constants. It is argued that $M_{\ell}(\infty)$ represents the most intermittent structures of passive scalars advected by turbulent flow. $\tilde{M}_{\ell}(\infty)$ represents the most intermittent structures of turbulent flow, which can be scaled as same as $\epsilon_{\ell}^{(\infty)}$. 
We assume that the joint moments of $\epsilon_{\ell}^{p}$ and $N_{\ell}^{q}$ can be scaled as $\left\langle N_{\ell}^{q} \epsilon_{\ell}^{p}\right\rangle \sim \ell^{\tau(q, p)}$, and that their limits scale as $M_{\ell}(\infty) \sim \ell^{-h_{T}}, \tilde{M}_{\ell}(\infty) \sim \ell^{-h}$, where $h_{T}$ and $h$ are the singular scaling exponents on the sheets and filaments of the highest intensity, respectively. From ( 8 ) and (9), one can obtain the following system of partial difference equations for $\tau(q, p)$,

$$
\begin{aligned}
& \tau(q+2, p)-\left(1+\beta_{T}\right) \tau(q+1, p)+\beta_{T} \tau(q, p) \\
& \quad=-h_{T}\left(1-\beta_{T}\right), \\
& \tau(q, p+2)-(1+\beta) \tau(q, p+1)+\beta \tau(q, p) \\
& \quad=-h_{h}(1-\beta) .
\end{aligned}
$$

Introducing the boundary conditions, $\tau(0,0)=$ $\tau(1,0)=\tau(0,1)=0$, we can obtain the solution of Eqs. (10),

$$
\begin{gathered}
\tau(q, p)=c\left(1-\beta^{p}\right)\left(1-\beta_{T}^{q}\right)+\frac{h}{1-\beta}\left(1-\beta^{\prime}\right) \\
+\frac{h_{T}}{1-\beta_{T}}\left(1-\beta_{T}^{q}\right)-h p-h_{T} q
\end{gathered}
$$

where $c$ is a coupling constant. Taking $a=h /(1-$ $\beta)+c, a_{T}=h_{T} /\left(1-\beta_{T}\right)+c$ and $b=-c$, we can rewrite the solution (11) as follows,

$$
\begin{aligned}
& \tau(q, p)=-h p-h_{T} q \\
& \quad+\left(a+a_{T}+b-a \beta^{p}-a_{T} \beta_{T}^{q}-b \beta^{p} \beta_{T}^{q}\right) .
\end{aligned}
$$

The expression in parentheses in (12) is the generating function for a bivariate Poisson distribution (see the discussion in next paragraph), $a$ and $a_{T}$ are the variances of $\epsilon_{\ell}$ and $N_{\ell}$, respectively, and $b$ is a constant representing the correlation between $\epsilon_{\ell}$ and $N_{\ell}$. For determining these parameters, see the discussions in $[14]$. Assuming $\left\langle\left(\delta T_{\ell}\right)^{n}\right\rangle \sim \ell^{t_{n}}$, we obtain an analytical formula for the scaling exponents of the $n$ thorder passive scalar structure functions by using (5) and (12).

$$
\begin{aligned}
z_{n 1} & =\frac{1}{3} n+\tau\left(\frac{1}{2} n,-\frac{1}{6} n\right) \\
& =\left(\frac{1}{3}+\frac{1}{6} h-\frac{1}{2} h_{T}\right) n+a\left(1-\beta^{-n / 6}\right) \\
& +a_{T}\left(1-\beta_{T}^{n / 2}\right)+b\left(1-\beta^{-n / 6} \beta_{T}^{n / 2}\right) .
\end{aligned}
$$

With the parameters given in Ref. [14], Eq. (13) can be written as

$$
\begin{aligned}
z_{n} & =3-\frac{n}{36}-2\left(\frac{3}{4}\right)^{n / 6}-\left(\frac{1}{2}\right)^{n / 2} \\
& +\gamma\left[1-\left(\frac{3}{4}\right)^{n / 6}-\left(\frac{1}{2}\right)^{n / 2}+\left(\frac{3}{4}\right)^{n / 6}\left(\frac{1}{2}\right)^{n / 2}\right],
\end{aligned}
$$

where $\gamma$ is a real positive number representing the correlation of $\epsilon_{\ell}$ and $N_{\ell}$. A good fit between (14) and measurements can be carried out for small $\gamma[14]$.

The bivariate Poisson distribution of both passive scalars and velocity can be formulated by assuming a two-dimensional cascade processes with infinite divisibility. The generating function of such a twodimensional infinitely divisible process [16] can be expressed as

$$
\begin{array}{r}
G\left(t_{1}, t_{2}\right)=\int_{-\infty}^{\infty} \int_{-\infty}^{\infty}\left[\operatorname{expi}\left(t_{1} x+t_{2} y\right)-1\right. \\
\left.-\mathrm{i}\left(t_{1}, x+t_{2} y\right)\right] /\left(x^{2}+y^{2}\right) \mathrm{d} K(x, y),
\end{array}
$$

where the kernel $K(x, y)$ is a non-decreasing function of bounded variation. If $K(x, y)$ is chosen

$$
\begin{aligned}
& \mathrm{d} K(x, y)=[-2 b \delta(x-1, y-1)-a \delta(x-1, y) \\
& \left.-a_{T} \delta(x, y-1)\right] \mathrm{d} x \mathrm{~d} y
\end{aligned}
$$

then

$G\left(s_{1}, s_{2}\right)=a+a_{T}+b-a s_{1}-a_{T} s_{2}-b s_{:} s_{2}$,

where $\delta(x, y)=\delta(x) \delta(y)$ is the Dirac function, and $s_{1}=\exp \left(\mathrm{i} t_{1}\right), s_{2}=\exp \left(\mathrm{i} t_{2}\right)$.

If $s_{1}$ and $s_{2}$ are replaced by $\beta^{\prime \prime}$ and $\beta^{q}$, respectively, the generation function (17) becomes the expression in parentheses in (12). Noting that the two-dimensional cascade process here is referred as $\left(N_{f} / N_{\ell}(\infty), \epsilon_{\ell} / \epsilon_{f}(\infty)\right)$, we can re-formulate the scaling exponents of the joint moments, which is the same as the Eq. (12). We remark that the twodimensional infinitively divisible process for the joint PDF of the scalar and velocity dissipation is realized only by choosing the kernel in the integral in (15). In the turbulent case, the kernel has one single "atom" [13], which has a log-Poisson distribution. In the case of a passive scalar advected by turbulent flow, the kernel must contain three "atoms" resulting in a bivariate log-Poisson distribution.

In summary, the bivariate Poisson distribution for both scalar dissipation, $N_{\ell}$, and turbulent dissipation, $\epsilon_{\ell}$, is formulated using the postulated hierarchical relations. We assume that not only the moments of the 
scalar dissipation and the velocity dissipation admit, respectively, the corresponding hierarchical relations, but also that their joint moments admit the extended hierarchical relations. In the extended hierarchical relations, the ratio constants are assumed to be associated with the most intermittent structures of dissipations. These two assumptions are sufficient to specify completely the scalings of the joint moments of $N_{\ell}$ and $\epsilon_{\ell}$. Furthermore, we have shown that the bivariate Poisson distribution is a realization of the two-dimensional random process of infinite divisibility, whose kcrnel has three atoms $[13,16]$. Given the bivariate Poisson distribution, the scaling exponents of the passive scalar structure function can be easily obtained [14] using the Obukhov and Corrsin RSH [3].

\section{References}

[1] M. Lesieur, Turbulence in Fluids, 2nd edition (Kluwer, Dordrecht, 1990).

[2] A.N. Kolmogorov, C.R. Acad. Sci. 30 (1941) 301.
[3] A.M. Obukhov, Izv. Akad. Nauk SSSR, Ser. Geogr. Geofiz. 13 (1949) 58;

S. Corrsin, J. Appl, Phys. 22 (1951) 469.

[4] U. Frisch, Turbulence: The Legacy of A.N. Kolmogorov (Cambridge Univ. Press, Cambridge, 1995).

[5] C. Meneveau, K.R. Sreenivasan, P. Kailasnath, M.S. Fan, Phys. Rev. A 41 (1990) 894.

[6] G. Ruiz-Chavarria, C. Baudet, S. Ciliberto, Physica D 99 (1996) 369.

[7] K.R. Sreenivasan, R.A. Antonia, Annu. Rev. Fluid Mech. 29 (1997) 435.

[8] A. Pumir, Phys. Fluids 6 (1994) 3974.

[9] S.Y. Chen, N.Z. Cao, Phys. Rev. Lett. 78 (1997) 3459.

[10] A.N. Kolmogorov, J. Fluid Mech. 13 (1962) 82.

[11] G. Stolovizky, P. Kailasnath, K.R. Sreenivasan, J. Fluid Mech. 297 (1995) 275.

[12] C.W. van Atta, Phys. Fluids 14 (1971) 1803.

[13] Z.S. She, E. Leveque, Phys. Rev. Lett. 72 (1994) 336; Z.S. She, E.C. Waymire, Phys. Rev. Lett. 74 (1995) 262.

[14] N.Z. Cao, S.Y. Chen, Phys. Fluids 9 (1997) 1203.

[15] E.D. Siggia, J. Fluid Mech. 107 (1981) 375; R.M. Kerr, J. Fluid Mech. 153 (1985) 31;

Z.S. She, E. Jackson, S.A. Orszage, Nature, 344 ( 1990) 226; A. Vincent, M. Meneguzzi, J. Fluid Mech. 225 (1991) 107.

[16] W. Feller, An Introduction to Probability Theory and its Application (II) (Wiley, New York, 1968). 